Self-mutual help group was the most appreciated free service, in which participants shared personal stories and perspectives thoughtfully and courageously. The training initiatives organized in collaboration with physicians helped them to learn tips for a better lifestyle management, diet and exercise, and psychosocial techniques but above all helped to overcame concerns and frustration regarding the lack of understanding in the medical community. The network succeeds to increased awareness and understanding of FMS across the public opinion and GPs.

References:

[1] Author: S.Mingolla, APMARR Project Manager; Co-authors: A.Celano, APMARR President; I. Cinieri, Psychologist, A. Marsico, Rheumatologist Disclosure of Interests: None declared

DOI: 10.1136/annrheumdis-2020-eular.1782

\section{OP0321-HPR HIGHER QUALITY OF CARE AND LESS SURGERY AFTER IMPLEMENTING OSTEOARTHRITIS GUIDELINES IN PRIMARY CARE- LONG-TERM RESULTS FROM A CLUSTER RANDOMIZED CONTROLLED TRIAL}

N. Osteras ${ }^{1}$, T. Moseng ${ }^{1}$, L. Van Bodegom-Vos ${ }^{2}$, K. Dziedzic ${ }^{3}$, Ø. Andreassen ${ }^{1}$, A. M. Fenstad ${ }^{4}$, O. Furnes ${ }^{4}$, J. Nygren Hansen ${ }^{1}$, B. Natvig ${ }^{5}$, J. H. Røtterud ${ }^{6}$, U. B. Schjervheim ${ }^{7}$, T. P. M. Vliet Vlieland ${ }^{2}$, K. B. Hagen ${ }^{1}{ }^{1}$ Diakonhjemmet Hospital, Oslo, Norway; ${ }^{2}$ Leiden University, Leiden, Netherlands; ${ }^{3}$ Keele University, Keele, United Kingdom; ${ }^{4}$ Haukeland University Hospital, Bergen, Norway; ${ }^{5}$ University of Oslo, Oslo, Norway; ${ }^{6}$ Akershus University Hospital, Lørenskog, Norway; ${ }^{7}$ Nes Municipality, Nes, Norway

Background: To improve quality of care for patients with hip and knee osteoarthritis (OA), a structured model for integrated OA care was developed and implemented among general practitioners (GPs) and physiotherapists (PTs) in primary care. The model was developed based on international treatment recommendations. After 6 months, patient-reported quality of care and satisfaction with care were greater, more patients were referred to physiotherapy and fewer to orthopaedic surgeon, and more patients fulfilled physical activity criteria among OA patients receiving the new model of care compared to the usual care control group ${ }^{1}$.

Objectives: To assess the long-term effects 12 months after implementing the model in primary care.

Methods: A cluster-randomised controlled trial with a stepped-wedge design was conducted in six Norwegian municipalities (clusters). The intervention included implementation of the model, facilitated by interactive workshops for GPs and PTs. The main components of the model were a PT led, 3 hour patient education programme followed by 8-12 weeks of individually tailored, supervised exercise. Patient participants were $\geq 45$ years with symptomatic hip or knee OA. Primary outcome was patient-reported quality of care (OsteoArthritis Quality Indicator questionnaire; $0-100,100=$ optimal quality). Secondary outcomes included satisfaction with care, referrals to physiotherapy, orthopaedic surgeon and magnetic resonance imaging (MRI), joint replacement surgery, fulfilment of physical activity recommendations, and proportion with overweight (body mass index $\geq 25 \mathrm{~kg} / \mathrm{m} 2$ ). Data was analysed using multilevel mixed models adjusted for age, sex and secular time.

Results: In all, 40 of 80 GPs and 37 of 64 PTs attended the workshops. A total of 393 patients with hip and knee OA were included, with 284 in the intervention and 109 in the usual care control group. In the intervention group, 92\% attended the OA education programme and $64 \%$ completed $\geq 8$ weeks of exercise. At 12 months the intervention group reported significantly higher quality of care (score 58 vs. 41 , mean difference: $17.6 ; 95 \% \mathrm{Cl} 11.1,24.0$ ) compared to the control group. The intervention group reported significantly higher satisfaction with care (Odds ratio (OR) $7.8 ; 95 \% \mathrm{Cl} 3.55,17.27$ ) and a significantly larger proportion (OR: $4.0 ; 95 \% \mathrm{Cl} 1.27,12.63$ ) met the recommendations for physical activity compared to the control group. A smaller proportion was referred to orthopaedic surgeon (OR $0.5 ; 95 \% \mathrm{Cl} 0.29,1.00)$ and a smaller proportion received joint replacement surgery in the intervention (4\%) compared to the control group (11\%) (OR $0.3 ; 95 \% \mathrm{Cl} 0.14,0.74)$. The proportion of patients referred to physiotherapy or MRI and the proportion with overweight were similar between the groups.

Conclusion: Implementation of a structured model for OA care led to improved quality of care, higher satisfaction with care and higher physical activity levels after 12 months. These results are comparable to the 6 months results, which indicate a long-term persistence in the beneficial effects of the intervention. The lower surgical rate in the intervention compared to the control group suggests that higher uptake of OA recommendations in primary care may reduce or postpone the need for surgery in people with hip or knee OA.

\section{References:}

[1] Østerås N, Moseng T, Bodegom-Vos LV, et al. Implementing a structured model for osteoarthritis care in primary healthcare: A stepped-wedge cluster-randomised trial. PLOS Medicine. 2019;16(10):e1002949.

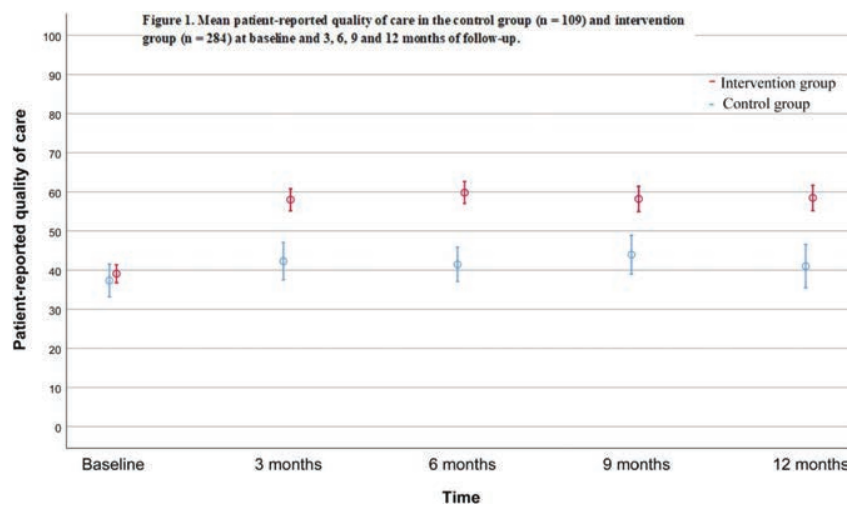

Disclosure of Interests: None declared

DOI: 10.1136/annrheumdis-2020-eular.3575

\section{How to communicate effectively with the patients}

\section{OP0322-PARE HOW TO COMMUNICATE DIAGNOSTIC INFORMATION AND CUTTING EDGE SCIENCE TO PATIENTS WITH RHEUMATIC DISEASES}

I. Loell ${ }^{1}$, S. Jansson ${ }^{1}$, J. Hammarström ${ }^{1}$, L. Alemo Munters ${ }^{1,2} .{ }^{1}$ Swedish Rheumatism Association, Stockholm, Sweden; ${ }^{2}$ Karolinska University Hospital, Functional Area Occupational Therapy and Physical Therapy, Stockholm, Sweden

Background: According to the 2017 Swedish Rheumatology Association (SRA) member strategy a recurring member survey as well as a member withdrawal survey was stipulated. The strategy was developed to evaluate to what extent SRA fulfills the requirements and expectations of its members. According to the 2019 survey, the most important output from a SRA membership, the members rank information about their diagnosis (\#1) and supporting the research of these diagnoses (\#2) most valuable.

Objectives: To transfer the medical and scientific expertise of the rheumatic diagnoses into lay information in order to meet the member's needs; to take part of the results of the cutting edge science and research progress, funded by SRA, that are relevant and important to individuals living with rheumatic conditions.

Methods: A targeted scientific communication strategy was made consisting of lectures, interviews and scientific writing created for multi-channel distribution.

Results: Actions taken upon the survey result

- Brief summaries of every research project funded by SRA in 2019 was written and distributed via social media.

- A research day for lay people was arranged in collaboration with a regional SRA branch and invited speakers. The filmed lectures are also available online.

- A research report with in-depth interviews with researchers and brief summaries about the research funded by SRA was produced. The report was printed and distributed in 70,000 copies to the SRA members, donors and at SRA meetings and conferences.

- Diagnosis sheets aimed to newly diagnosed patients with the most essential information has been developed in collaboration with a patient research partner and an expert researcher within the field. The sheet is printable and can be distributed by any healthcare practitioner or by patients/public.

- Online patient school prototype - gout. In collaboration with the SRA funded gout network we are gathering high quality information about the diagnosis, treatment, self-care and support in the meeting with the healthcare provider for patients to easily navigate and to find robust answers to their inquiries about their disease.

Conclusion: As a member of SRA, regardless of rheumatic disease, the main interest is knowing more about their diagnosis and about the ongoing research in the field. Through collaborations and communicating rheumatic conditions and 
research within the field in lay language the patients can experience self-empowerment and the need for patient education can be met.

Disclosure of Interests: None declared

DOI: 10.1136/annrheumdis-2020-eular.3040

\section{The cost-opportunity of screening: osteoporosis in the general population}

\section{OP0323 INCIDENCE OF CLINICAL FRAGILITY FRACTURES IN POSTMENOPAUSAL WOMEN WITH RHEUMATOID ARTHRITIS. A MULTICENTRIC CASE-CONTROL STUDY}

C. Gómez Vaquero ${ }^{1}$, J. M. Olmos ${ }^{2}$, J. L. Hernández ${ }^{2}$, D. Cerda ${ }^{3}$, C. Hidalgo ${ }^{4}$, J. Martínez López ${ }^{5}$, L. M. Arboleya Rodríguez ${ }^{6}$, J. Aguilar del Rey ${ }^{7}$, S. Martinez Pardo $^{8}$, I. Ros ${ }^{9}$, X. Surís ${ }^{10}$, D. Grados Canovas ${ }^{11}$, C. Beltrán Audera ${ }^{12}$, E. Suero-Rosario ${ }^{13}$, I. Gómez Gracia ${ }^{14}$, A. Salmoral ${ }^{14}$, I. Martín-Esteve ${ }^{13}$, H. Florez ${ }^{15}$, A. Naranjo ${ }^{16}$, S. Castañeda ${ }^{17}$, S. Ojeda ${ }^{16}$, S. García Carazo ${ }^{18}$ A. García-Vadillo ${ }^{17}$, L. López Vives ${ }^{19}$, À. Martínez-Ferrer ${ }^{20}$, H. Borrell Paños ${ }^{19}$, P. Aguado ${ }^{18}$, R. Castellanos-Moreira ${ }^{15}$, C. Tebé ${ }^{21}, \mathrm{~N}$. Guañabens ${ }^{15}$ on behalf of Grupo de Trabajo OsteoResSER de la Sociedad Española de Reumatología. ${ }^{1}$ Hospital Universitari de Bellvitge, L'Hospitalet de Llobregat, Spain; ${ }^{2}$ Hospital Marqués de Valdecilla, Medicina Interna, Santander, Spain; ${ }^{3}$ Hospital Sant Joan Despí Moisès Broggi, Sant Joan Despí, Spain; ${ }^{4}$ Hospital Universitario de Salamanca, Salamanca, Spain; ${ }^{5}$ Hospital Universitario Fundación Jiménez Díaz, Madrid, Spain; ${ }^{6}$ Hospital Universitario Central de Astúrias, Oviedo, Spain; ${ }^{7}$ Hospital Universitario Virgen de la Victoria, Málaga, Spain; ${ }^{8}$ Hospital Universitario Mútua de Terrassa, Terrassa, Spain; ${ }^{9}$ Hospital Son Llàtzer, Palma, Spain; ${ }^{10}$ Hospital General de Granollers, Granollers, Spain; ${ }^{11}$ Hospital d'Igualada, Igualada, Spain; ${ }^{12}$ Hospital Universitario Miguel Servet, Zaragoza, Spain; ${ }^{13}$ Hospital General Mateu Orfila, Maó-Menorca, Spain; ${ }^{14}$ Hospital Universitario Reina Sofía, Córdoba, Spain; ${ }^{15}$ Hospital Clínic de Barcelona, Barcelona, Spain; ${ }^{16}$ Hospital Universitario de Gran Canaria Dr. Negrín, Las Palmas de Gran Canaria, Spain; ${ }^{17}$ Hospital Universitario de La Princesa, Madrid, Spain; ${ }^{18}$ Hospital Universitario La Paz, Madrid, Spain; ${ }^{19}$ Hospital de Sant Rafael, Barcelona, Spain; ${ }^{20}$ Hospital Universitario Doctor Peset, Valencia, Spain; ${ }^{21}$ Servicio de Asesoría en Estadística, Institut d'Investigació Biomèdica de Bellvitge-IDIBELL, L'Hospitalet de Llobregat, Spain

Background: Incidence of clinical fractures in rheumatoid arthritis (RA) is not as well-known as hip or vertebral fracture incidence.

Objectives: 1 . To estimate the incidence of clinical fragility fractures in a population of postmenopausal women diagnosed with RA and compare it with that of the general population; 2 . To analyze the risk factors for fracture.

Methods: 330 postmenopausal women with RA from 19 Spanish Rheumatology Departments, randomly selected from the registry of RA patients in each center. The control group consisted of 660 Spanish postmenopausal women from the Camargo Cohort. Clinical fractures during the previous 5 years were recorded. Assessed risk factors for fracture were: sociodemographic characteristics, BMD and variables related to $R A$.

Results: Median age of RA patients was 64 yrs. vs. 63 yrs. in controls (ns). Evolution of the disease was $8 \mathrm{yrs} .78 \%$ and $76 \%$ had RF and ACPA+, respectively. $69 \%$ of patients were in remission or low activity. $85 \%$ had received glucocorticoids and methotrexate and $40 \%$ at least one biological DMARD. We identified 105 fractures (87 fragility and 18 traumatic) in 75 patients. Fifty-four patients and 47 controls had at least one major fracture (MF) $(p<0.001)$. Incidence of MF was 3.55 per 100 patient-year in patients and 0.72 in controls. Risk factors for MF in RA patients were age, previous fracture, parental hip fracture, postmenopausal period, hip BMD and cumulative dose of glucocorticoids. In controls, risk factors were age, age at menopause and lumbar BMD.

Among RA-associated factors, MFs were associated with erosions, disease activity and disability. Previous fracture in RA patients was a strong risk for MF (HR: 10.37 [95\% Cl: 2.95-36.41]).

Conclusion: Between 3 and 4 of every 100 postmenopausal women with RA have a major fracture per year, four times more than the general population. Disease activity and disability associated with RA, the cumulative dose of glucocorticoids and mainly previous fracture are associated with the development of fragility fractures.

References: None

Acknowledgments: Funded in part by ISCIII (PI18/00762) that included FEDER funds from the EU.

Disclosure of Interests: Carmen Gómez Vaquero: None declared, Jose Manuel Olmos: None declared, J. Luis Hernández: None declared, Dacia Cerda: None declared, Cristina Hidalgo: None declared, JA Martínez López: None declared,
Luis Marcelino Arboleya Rodríguez: None declared, Javier Aguilar del Rey: None declared, Silvia Martinez Pardo: None declared, Inmaculada Ros: None declared, Xavier Surís: None declared, Dolors Grados Canovas: None declared, Chesús Beltrán Audera: None declared, Evelyn Suero-Rosario: None declared, Inmaculada Gómez Gracia: None declared, Asunción Salmoral: None declared, Irene Martín-Esteve: None declared, Helena Florez: None declared, Antonio Naranjo Grant/research support from: amgen, Consultant of: UCB, Speakers bureau: AMGEN, Santos Castañeda: None declared, Soledad Ojeda Speakers bureau: AMGEN, LILLY, GEBRO, S García Carazo: None declared, Alberto García-Vadillo: None declared, Laura López Vives: None declared, À Martínez-Ferrer: None declared, Helena Borrell Paños: None declared, Pilar Aguado: None declared, Raul Castellanos-Moreira: None declared, Cristian Tebé: None declared, Núria Guañabens: None declared DOI: 10.1136/annrheumdis-2020-eular.1680

\section{OP0324 CLUSTERING OF FRAGILITY FRACTURES BY SITE IN PATIENTS REFERRED FOR BONE MINERAL DENSITY ESTIMATION: AN OBSERVATIONAL STUDY}

M. Dey ${ }^{1,2}$, M. Bukhari ${ }^{3}{ }^{1}$ Institute of Ageing and Chronic Disease, University of Liverpool, Musculoskeletal Biology I, Liverpool, United Kingdom; ${ }^{2}$ Aintree University Hospital, Liverpool University Hospitals NHS Foundation Trust, Academic Rheumatology, Liverpool, United Kingdom; ${ }^{3}$ Royal Lancaster Infirmary, University Hospitals of Morecambe Bay NHS Foundation Trust, Rheumatology, Lancaster, United Kingdom

Background: Fragility fractures (FF) are those resulting from mechanical forces equivalent to a fall from standing height or less [1]. They most commonly occur in the spine (vertebrae), forearm, and femur, but also occur at other sites. Prevalence markedly increases with age, due to age-related and menopause-related bone loss. FF cause substantial pain and disability, and are associated with decreased life expectancy. While many studies have investigated risk factors associated with FF, there are few data on the association between FF sites in at-risk patients.

Objectives:

1. Establish the most common sites of $\mathrm{FF}$ in patients presenting for bone mineral density (BMD) estimation.

2. Identify patterns of co-existing FF in the above cohort by applying cluster analysis.

Methods: We retrospectively reviewed the clinical records of 28868 patients presenting for BMD estimation at a district general hospital in North West England, 2004-2016, identifying those who had sustained one or more FF. Site(s) of FF were recorded for each patient, categorised as: ankle, elbow, femur, forearm, humerus, pelvis, ribs, spine, tibia or fibula (recorded as "tibfib"). Cluster analysis was performed on fracture sites, using Jaccard similarity coefficient. Results were plotted on a dendrogram and divided into clusters, as per results derived from elbow and silhouette cluster methods.

Results: Out of 28868 patients presenting for BMD estimation, 11003 were identified as having sustained one or more FF. $84.6 \%$ patients were female, with overall mean age $67.5 y e a r s$ and median T-score -1.12 SD. The most common site of FF was the forearm $(n=5045)$, most commonly co-existing with fractures of the tibia or fibula. Frequencies of the most common and co-existing FF sites are shown in Figure 1 (top). Cluster analysis identified 3 clusters: ankle and elbow; forearm, tibia/fibula, ribs, and spine; pelvis, femur, and humerus. The second half of Figure 1 displays the dendrogram of cluster analysis results, with Jaccard similarity measure.

Conclusion: We applied cluster analysis to a large cohort of patients presenting for BMD estimation. Our results are in keeping with previous studies demonstrating the FF to most commonly occur in the forearm, and in those with osteopenia (T-score $-2.5<-1 \mathrm{SD}$ ) [2]. To our knowledge, this is the first study to apply cluster analysis to sites of FF. Results may be due to differences in cortical and trabecular bone structure, and have potential to aid prevention, monitoring, and management in at-risk patients.

References:

[1] National Institute for Health and Care Excellence (NICE). Osteoporosis : assessing the risk of fragility fracture - Clinical Guideline (CG146). 2012;(August):1-14.

[2] Siris ES et al. Bone mineral density thresholds for pharmacological intervention to prevent fractures. Arch Intern Med. 2004 May 24;164(10):1108-12.

Disclosure of Interests: Mrinalini Dey: None declared, Marwan Bukhari Speakers bureau: Bristol-Myers Squib, UCB celltech, Roche/Chugai, Pfizer, Abbvie, Merck, Mennarini, Sanofi-aventis, Eli-Lilly, Janssen, Amgen and Novartis. DOI: 10.1136/annrheumdis-2020-eular.856 